escape into the auditory canal. Should this occur, the injection must be repeated two or three times until the covering of the superior wall bulges downward, showing that sufficient fluid is retained.

The syringe is cautiously withdrawn and a small pledget of cotton saturated with tonogen is pressed against the ficld of operation. After waiting from ten to fifteen minutes to allow the anesthetic to take effect, the operation can be performed without the slightest inconvenience to the patient.

On the completion of the operation, the field is dusted with a mixture of equal parts of anesthesin and boracic acid to counteract the severe pain which would otherwise develop in an hour or two following the operation. This indication is fully met by this powder, as in the cases where it has been used the patients have not complained of any pain subsequent to the operation.

The syringe used for these injections is of metal, holding 1 c.c.; and with a needle modified by Neumann. A pair of half rings (which are detachable) have been added to the syringe to facilitate the injection.

In conclusion, we wish to express our gratitude to Professor Politzer and his assistants for the exceptional opportunities afforded us for study during our term of service in the clinic at Vienna.

\section{PUS TUBES IN THE MALE, AND THEIR SUR- GICAL TREATMENT.}

W. T. BELFIELD, M.D.

Associate Professor of Surgery, Rush Medical College. CHICAGO.

Due attention is not generally accorded to the fact that the genital duct proper in the male-seminal vesicle, vas deferens and epididymis-is just as prone to suppuration as is the urethra. Suppuration in the vesicle, it is true, has been recognized, in recent years, as a frequent feature of gonorrhea; but the extension of this infection to the epididymis-frequent and familiar though it be-is not generally considered a suppurating process. Even the best of our modern treatises expressly state that suppuration is exceptional in epididymitis, gonorrheal or other; they consistently prescribe the usual medical treatment, but do not advise the evacuation of pus, except in those rare cases in which fluctuation becomes distinct.

The pus infections of the epididymis and of the vas deferens present a complete though neglected analogy with the familiar pus infections of the fallopian tubes. An epididymitis following prostatic pus infection, whether gonorrheal or other, is usually suppurative; pus tubes are quite as common in the male as in the female; hence the rational treatment of such acute epididymitis should frequently be surgical, i. e., it should include the evacuation of pus by incision.

In the mildest cases such incision may be needlessiy radical, since the pus may presently find the natural channel into the urethra; on the other hand, the acute hydrocele and the scrotal edema, which so often accompany severe acute epididymitis, are infallible signs of suppuration in the epididymis, and the incision is followed by relief from pain and subsidence of the swelling. Even when no pus escapes, the relief of tension affords marked comfort and insures a relatively speedy convalescence.

If direct exit for the pus by incision into the epi-didymis be not provided, the gathering pus often bur- rows along the vas into the urethra; and thus is explained the familiar phenomenon that decrease of the pain of epididymitis is accompanied by marked increase in the discharge from the meatus. Moreover, persistent suppuration in the epididymis may feed the gleet which follows, just as pus-tubes in the female feed a leucorrhea; and this gleet may recur indefinitely, in spite of elaborate urethral and internal medication, until the hard and tender swelling of the epididymis is incised and the contained pus evacuated.

In other words, the successful treatment of a gleet sometimes requires incision into the epididymis.

Again, chronic epididymitis through pus infection may require surgical treatment; persistent pain and tenderness following the acute infection (sometimes miscalled neuralgia of the testis) is relieved by incision or excision of the epididymal pus focus; destruction of the testis by extension of the pus infection from the epididymis may be prevented by the same treatment.

The lower end or tail of the epididymis presents the chief swelling in acute or chronic epididymitis (pus infection), since in this tail is coiled the greater portion of the 20 feet of the epididymal tube; and into this lower end the incision usually should be made. Simple puncture with bistoury or aspirating needle may sometimes suffice; yet drainage is better assured by an incision a half inch or more long, the edges of the pus cavity being loosely stitched to the cut edges of the skin, and a small drain inserted.

In a few cases $I$ have practiced a therapeutic measure which, according to my present information, is novel, namely, injections into the vas deferens and seminal vesicle, through a needle introduced into the vas just above the epididymis. As the dilated upper extremity of the vas, the ampulla, bears about the same relation to the vesicle that the auricle does to the ventricle of the heart, a liquid injected into the vas easily reaches the vesicle; this can be clearly demonstrated by injecting Prussian blue in water into the vas of the fresh subject.

The therapeutic value of such injection is not yet accurately determined; at present it can be merely affirmed that this is a practicable way-and as yet the only way-for directly medicating the male genital tube, including the vesicle, which is so commonly and so persistently infected by various pus bacteria and by the gonococcus.

\section{Special Article}

\section{M M U N I T Y. CHAPTER XIII.}

THE BACTERICIDAL PROPERTY OF SERUMS.

There are two theories of note which concern the ability of the body to destroy bacteria. One of these is the phagocytic theory of Metchnikoff, according to which The Cellular micro-organisms can not be destroyed withand Humoral out the direct or indirect participation of Theories. the phagocytic cells; this is the cellular theory. The second, the humoral theory, supposes that the power resides in the fluids of the body alone; in its improved form it now holds that antibacterial immunity should be considered cellulo-humoral, and that whatever bactericidal power the body fluids have is derived primarily from the body cells. Many who appreciate the action of the serum, however, also believe that phagocytic cells may take up and, in certain instances, destroy micro-organisms; this combined view seems best to fit the facts as known at present. 